\title{
THEORETICAL LIMITS ON THE PROPERTIES OF \\ LOW-VELOCITY M-DWARFS
}

\author{
P. BIERMANN
}

Universitäts-Sternwarte Göttingen, F.R.G.

\begin{abstract}
Using a version of the Toomre-criterion (Toomre, 1964) for a mixture of stellar populations, limits are derived for the kinematical properties of the newly discovered population of low-velocity M-dwarfs (Weistrop, 1972; Murray and Sanduleak, 1972); these stars are claimed to be a large massfraction ( $\gtrsim 0.5$ ) of all stars in the solar neighbourhood and to have only $10 \mathrm{~km} \mathrm{~s}^{-1}$ velocity dispersion. They could make up for the locally missing mass (Oort, 1965; Schmidt, 1974). It is found that a configuration of stellar populations with the M-dwarfs included (having such properties) is not stable in the sense of the Toomre-criterion. The limiting stability properties are given.
\end{abstract}

\section{Introduction}

Using the method of moments of the Boltzmann-equation the Toomre-criterion has been rederived. Since this method leads to an infinite set of equations, cutoff conditions have to be imposed. The cutoff conditions assumed here are: (a) The temperature-asymmetry-tensor of the stellar motions (the tensor of third order) is identically zero. (b) All higher order tensor-moments are ignored. This blunt approach reproduces within $10 \%$ the limiting velocity dispersion of stellar motions for stability against axisymmetric perturbations in a flat disk; since the numbers that go into this number, the surface mass density and the epicyclic frequency, are not known to that accuracy, the difference is not significant.

The moment-method has been used by King (1965), Larson (1969), Hunter (1970), and many others. Here the criterion is generalized to many stellar populations and applied to the problem of the low-velocity M-dwarfs, in Sections 2 and 3, respectively. The results are discussed in Section 4. Superpositions of stellar systems in flat galaxies have previously been considered by Vandervoort (1970).

We assume here that the low-velocity M-dwarfs are a general population in the disk of the galaxy, and that their properties in the solar neighbourhood reflect those at large.

\section{The Criterion}

We define: $k$ is the wave-number of the perturbation, $\alpha_{j}$ is the radial component of the temperature-tensor of stellar motions (the $r-r$-component) of the $j$ stellar populations, $\kappa$ the epicyclic frequency, $\varrho_{j_{0}}$ the unperturbed surface density of the $j$ stellar population, $\varrho_{t_{0}}$ the total unperturbed stellar surface density, and $G$ the gravitational constant. Then the critical wave-number is

$$
k_{\mathrm{crit}}=\frac{\kappa^{2}}{2 \pi G \varrho_{t_{0}}} .
$$


Defining

$$
x=\frac{k_{\mathrm{crit}}}{k} \text { and } y_{j}=\frac{\alpha_{j} k_{\mathrm{crit}}^{2}}{\kappa^{2}}
$$

one finds (for one stellar population only) an expression relating the velocity dispersion to the wavelength separating stable and unstable regions:

$$
\left(3 \frac{y}{x^{2}}-\frac{1}{x}+1\right)\left(\frac{y}{x^{2}}+4\right)-9 \frac{y}{x^{2}}=0 \text {. }
$$

A related expression has been discussed by Hunter (1970). The maximum of the curve $y(x)$ gives the minimum that $y$ can have to ensure stability for all $x \in[0,1]$ :

$$
y_{\max }=0.2272 \text { at } x=0.60 \text {. }
$$

The corresponding number of Toomre (1964) is 0.2857 . Generalizing to $J$ stellar populations the relevant equation is:

$$
1=\sum_{j=1}^{J} \frac{\varrho_{j_{0}}}{\varrho_{t_{0}}} \frac{x}{3 y_{j}+x^{2}-\frac{9 y_{j} x^{2}}{y_{j}+4 x^{2}}} .
$$

\section{Results}

Wielen (1974) finds that the average velocity dispersion of all observed stars is 48 $\mathrm{km} \mathrm{s}^{-1}$. We adopt this number from Wielen and take from the model of the solar neighbourhood of Biermann and Tinsley (1974) the numbers for the mass fractions of the different stellar populations: 0.17 for normal stars, 0.26 for invisible remnants, and 0.57 for the low-velocity M-dwarfs. The normal stars and the invisible remnants are thus assumed to have $48 \mathrm{~km} \mathrm{~s}^{-1}$ velocity dispersion and the low-velocity $\mathrm{M}$ dwarfs $10 \mathrm{~km} \mathrm{~s}^{-1}$. Three questions can now be asked from the criterion:

(a) Is the configuration stable as given? For the answer we write down a necessary condition (index $j$ is for the M-dwarfs here)

$$
\frac{\varrho_{j_{0}}}{\varrho_{t_{0}}} \frac{x}{3 y_{j}+x^{2}-\frac{9 y_{j} x^{2}}{y_{j}+4 x^{2}}} \leqslant 1 \text { for all } x \in[0,1] \text {. }
$$

This expression corresponds to the assumptions that all populations except $j$ have infinite velocity dispersion. (In that extreme case it becomes a sufficient condition.) We find then that $\varrho_{j_{0}} / \varrho_{t_{0}} \leqslant 0.33$ and thus that the configuration as given is not stable.

(b) If we use all mass fractions and the velocity dispersions for the normal stars and the invisible remnants as adopted we can find the lowest allowable velocity dispersion for the low-velocity M-dwarfs: The answer is $21 \mathrm{~km} \mathrm{~s}^{-1}$. 
(c) If we use the velocity dispersions of all stellar populations as adopted, we can ask for the maximum mass fraction the low-velocity M-dwarfs can have: We find 0.30 .

\section{Discussion}

Applying the generalized Toomre-criterion we find

(a) the mixture of stellar populations with kinematical properties as claimed to be observed is not stable,

(b) the lowest allowable velocity dispersion for the low-velocity M-dwarfs (at 0.57 mass fraction) is $21 \mathrm{~km} \mathrm{~s}^{-1}$,

(c) the largest allowable mass fraction (at $10 \mathrm{~km} \mathrm{~s}^{-1}$ velocity dispersion) is 0.30 . Evidently, intermediate cases can be derived.

The observations are still discussed and extended (Schmidt, 1974; Thé and Staller, 1974). These M-dwarfs could be a local phenomenon and then some problems would disappear (Biermann, 1974).

The relevance of the Toomre-criterion is clearly limited (Miller, 1974); however, if it indicates instability, more detailed considerations of the same physics will not render a configuration stable, whereas the reverse cannot be said.

The form of the moment-method used here cannot be applied for non-axisymmetric perturbations. But it is a useful example of the application of the moment-method which may be helpful in further generalizations.

Putting gas into the equations just as another population would restrict the limits for the M-dwarfs even further. It remains an interesting task to really integrate over all stellar populations that can be distinguished kinematically.

\section{Acknowledgements}

I wish to thank Dr I. R. King, Dr M. Schmidt, Dr A. Toomre, and Dr R. Wielen for interesting discussions. This work was done while the author was supported by the Deutsche Forschungsgemeinschaft (Bi 191/1) at Columbia University and UC Berkeley. The visit at Berkeley was made possible through a generous invitation from $\mathrm{Dr}$ J. Silk. I wish to thank the departments at New York and Berkeley for their hospitality.

\section{References}

Biermann, P. and Tinsley, B. M.: 1974, Astron. Astrophys. 30, 1.

Biermann, P.: 1974, Astron. Astrophys. 30, 31.

Hunter, C. : 1970, Studies in Appl. Math. 49, 59.

King, I. R.: 1965, Astron. J. 70, 296.

Larson, R. B.: 1969, Monthly Notices Roy. Astron. Soc. 145, 405.

Miller, R. H.: 1974, Astrophys. J. 190, 539.

Murray, C. A. and Sanduleak, N.: 1972, Monthly Notices Roy. Astron. Soc. 157, 273.

Oort, J. H.: 1965, Stars and Stellar Systems 5, 455.

Schmidt, M.: 1974, this volume, p. 325.

Thé, P. S. and Staller, R. F. A.: 1974, Astron. Astrophys. (in press).

Toomre. A.: 1964, Astrophys. J. 139. 1217. 
Vandervoort, P. O.: 1970, Astrophys. J. 162, 453.

Weistrop, D.: 1972, Astron. J. 77, 849.

Wielen, R.: 1974, in G. Contopoulos (ed.), Highlights of Astronomy, Vol. 3, D. Reidel Publ. Co., Dordrecht - Holland, p. 395.

\section{DISCUSSION}

Miller: Do you mean that some fraction of high velocity stars such as we have in the solar neighborhood cannot stabilize in the sense of the Toomre criterion?

Biermann: Even for infinite velocity dispersion for all stars except the M-stars, a thin disk with $57 \%$ of the mass in $\mathrm{M}$ dwarfs and $10 \mathrm{~km} \mathrm{~s}^{-1}$ velocity dispersion is not stable. 